\title{
DISPERSIVE ESTIMATES FOR MANIFOLDS WITH ONE TRAPPED ORBIT
}

\author{
HANS CHRISTIANSON
}

\begin{abstract}
For a large class of complete, non-compact Riemannian manifolds, $(M, g)$, with boundary, we prove high energy resolvent estimates in the case where there is one trapped hyperbolic geodesic. As an application, we have the following local smoothing estimate for the Schrödinger propagator:

$$
\int_{0}^{T}\left\|\rho_{s} e^{i t\left(\Delta_{g}-V\right)} u_{0}\right\|_{H^{1 / 2-\epsilon}(M)}^{2} d t \leq C_{T}\left\|u_{0}\right\|_{L^{2}(M)}^{2}
$$

where $\rho_{s}(x) \in \mathcal{C}^{\infty}(M)$ satisfies $\rho_{s}=\left\langle\operatorname{dist}_{g}\left(x, x_{0}\right)\right\rangle^{-s}, s>\frac{1}{2}$, and $V \in \mathcal{C}^{\infty}(M)$, $0 \leq V \leq C$ satisfies $|\nabla V| \leq C\left\langle\text { dist }\left(x, x_{0}\right)\right\rangle^{-1-\delta}$ for some $\delta>0$. From the local smoothing estimate, we deduce a family of Strichartz-type estimates, which are used to prove two well-posedness results for the nonlinear Schrödinger equation.

As a second application, we prove the following exponential local energy decay estimate for solutions to the wave equation when $\operatorname{dim} M=n \geq 3$ is odd and $M$ is equal to $\mathbb{R}^{n}$ outside a compact set:

$$
\begin{aligned}
& \int_{M}\left|\psi \partial_{t} u\right|^{2}+|\psi \nabla u|^{2} d x \\
& \quad \leq C e^{-t / C}\left(\|u(x, 0)\|_{H^{1+\epsilon}(M)}^{2}+\left\|D_{t} u(x, 0)\right\|_{H^{\epsilon}(M)}^{2}\right),
\end{aligned}
$$

where $\psi \in \mathcal{C}^{\infty}(M), \psi \equiv e^{-|x|^{2}}$ outside a compact set.
\end{abstract}

\section{INTRODUCTION}

In this note we show how the results of Chr1, Chr2 on cutoff resolvent estimates near closed hyperbolic orbits can be combined with the non-trapping resolvent estimates in $\mathrm{CPV}$ to obtain resolvent bounds in the case of one trapped hyperbolic orbit with a logarithmic loss. As an application, we use the arguments of BGT2] to prove the following local smoothing theorem for the Schrödinger propagator.

Theorem 1. Suppose $(M, g)$ is a Riemannian manifold (with or without boundary) which satisfies the assumptions of the introduction, $\gamma \subset M$ is a closed hyperbolic geodesic, and $-\Delta_{g}$ is the Laplace-Beltrami operator with Dirichlet boundary conditions. Then for each $\epsilon>0$ there is a constant $C$ such that

$$
\int_{0}^{T}\left\|\rho_{s} e^{i t\left(\Delta_{g}-V(x)\right)} u_{0}\right\|_{H^{1 / 2-\epsilon}(M)}^{2} d t \leq C\left\|u_{0}\right\|_{L^{2}(M)}^{2},
$$

where $\rho_{s} \in \mathcal{C}^{\infty}(M)$ satisfies

$$
\rho_{s}(x) \equiv\left\langle d_{g}\left(x, x_{0}\right)\right\rangle^{-s}
$$

Key words and phrases. local smoothing, local energy decay, trapping geometry, semiclassical resolvent. 
for $x_{0}$ fixed and $x$ outside a compact set, and $V \in \mathcal{C}^{\infty}(M), 0 \leq V \leq C$ satisfies

$$
|\nabla V| \leq C\left\langle\operatorname{dist}\left(x, x_{0}\right)\right\rangle^{-1-\delta}
$$

for some $\delta>0$

Remark 1.1. We will see that in some cases the weighted resolvent has no poles on the real axis, and we can conclude the estimate (1.1) is global in time at the expense of replacing $\rho_{s}$ with super-exponentially decreasing weights. That is, in these cases we have

$$
\int_{0}^{\infty}\left\|\psi e^{i t\left(\Delta_{g}-V\right)} u_{0}\right\|_{H^{1 / 2-\epsilon}(M)}^{2} d t \leq C\left\|u_{0}\right\|_{L^{2}(M)}^{2},
$$

where $\psi \equiv \exp \left(-\operatorname{dist}_{g}\left(x, x_{0}\right)^{2}\right)$ outside a compact set. This is the case, for example, if $g$ is an asymptotically Euclidean scattering metric, and $\partial M=\emptyset$ (see [Mel, Theorem $3, \S 10])$. It is also the case if $(M, g)$ is equal to $\mathbb{R}^{n}$ outside a compact set, $n \geq 2$, and $V$ satisfies (1.6) below (see [Vai, Theorem 8, Ch.9]). See Remarks 2.1 and 3.2 .

As a second application, we study the wave equation on $(M, g)$ :

$$
\left\{\begin{array}{l}
\left(-D_{t}^{2}-\Delta_{g}+V(x)\right) u(x, t)=0, \quad(x, t) \in M \times[0, \infty) \\
u(x, 0)=u_{0} \in H^{1}(M), D_{t} u(x, 0)=u_{1} \in L^{2}(M),
\end{array}\right.
$$

where $-\Delta_{g}$ is the Dirichlet Laplace-Beltrami operator on functions and $V \in \mathcal{C}^{\infty}(M)$ satisfies

$$
\exp \left(-\operatorname{dist}_{g}\left(x, x_{0}\right)^{2}\right) V=o(1) .
$$

Let $\psi \in \mathcal{C}^{\infty}(M)$ satisfy

$$
\psi \equiv \exp \left(-\operatorname{dist}_{g}\left(x, x_{0}\right)^{2}\right)
$$

for $x$ outside a compact set and $x_{0}$ fixed. For $u$ satisfying (1.5), we define the local energy, $E_{\psi}(t)$, to be

$$
E_{\psi}(t)=\frac{1}{2}\left(\left\|\psi \partial_{t} u\right\|_{L^{2}(M)}^{2}+\|\psi u\|_{H^{1}(M)}^{2}\right) .
$$

Theorem 2. Suppose $(M, g)$ is equal to $\mathbb{R}^{n}$ outside a compact set, $n=\operatorname{dim} M \geq 3$ is odd, and $\gamma \subset M$ is a hyperbolic trapped ray with no other trapping. Then for each $\epsilon>0$ and each

$$
\begin{aligned}
& u_{0} \in \mathcal{C}_{c}^{\infty}(M) \cap H^{1+\epsilon}(M), \text { and } \\
& u_{1} \in \mathcal{C}_{c}^{\infty}(M) \cap H^{\epsilon}(M),
\end{aligned}
$$

there is a constant $C>0$ such that

$$
E_{\psi}(t) \leq C e^{-t / C}\left(\left\|u_{0}\right\|_{H^{1+\epsilon}(M)}^{2}+\left\|u_{1}\right\|_{H^{\epsilon}(M)}^{2}\right) .
$$

Here the constant $C$ depends only on $\epsilon>0, g, n, \psi$, and the support of $u_{0}$ and $u_{1}$.

Remark 1.2. The estimate (1.8) holds whenever the resolvent admits a meromorphic extension to $\mathbb{C}$ with no poles in a complex neighbourhood of an interval $[-C, C] \subset \mathbb{R}$, which holds also, for example, if $(M, g)$ is an exterior domain in $\mathbb{R}^{n}$ with $n \geq 3$ odd. 
The problem of "local smoothing" estimates for the Schrödinger equation has a long history. The sharpest results to date are those of Doi [Doi] and Burq Bur2]. Doi proved if $M$ is asymptotically Euclidean, then one has the estimate

$$
\int_{0}^{T}\left\|\psi e^{i t \Delta_{g}} u_{0}\right\|_{H^{1 / 2}(M)}^{2} d t \leq C\left\|u_{0}\right\|_{L^{2}(M)}^{2}
$$

for $\psi \in \mathcal{C}_{c}^{\infty}(M)$ if and only if there are no trapped sets. Burq's paper showed if there is trapping due to the presence of several convex obstacles in $\mathbb{R}^{n}$ satisfying certain assumptions, then one has the estimate (1.9) with the $H^{1 / 2}$ norm replaced by $H^{1 / 2-\epsilon}$ for $\epsilon>0$.

The estimates with the $\epsilon>0$ loss in trapping geometries corresponds to a logarithmic loss in resolvent estimates for these geometries (see Theorem 3). With more care, one could replace the $\epsilon>0$ loss in derivative with a logarithmic loss in derivative, which may help in certain applications. The proof of Theorem 3 uses a semiclassical reduction to consider an operator of the form

$$
P(h)-z=-h^{2} \Delta_{g}-z,
$$

with $z \in[E-\delta, E+\delta]+i\left(-c_{0} h, c_{0} h\right)$ for $E, \delta>0$. It is shown in Chr1, Chr2 that for $A \in \Psi_{h}^{0,0}(M)$ with sufficiently small wavefront set near $\gamma \subset\{p=E\}$,

$$
\|(P(h)-z) A u\|_{L^{2}} \geq C^{-1} \frac{h}{\log (1 / h)}\|A u\|_{L^{2}} .
$$

We will use the main results from [CPV] and propagation of singularities to extend this to an estimate on $M$.

As an application of Theorem 1 and the non-trapping Strichartz estimates of HTW, we study the nonlinear Schrödinger equation

$$
\left\{\begin{array}{c}
i \partial_{t} u+\left(\Delta_{g}-V(x)\right) u=F(u) \text { on } I \times M ; \\
u(0, x)=u_{0}(x),
\end{array}\right.
$$

where $I \subset \mathbb{R}$ is an interval containing 0 and $V \in \mathcal{C}_{c}^{\infty}(M), V \geq 0$. Here the nonlinearity $F$ satisfies

$$
F(u)=G^{\prime}\left(|u|^{2}\right) u,
$$

and $G: \mathbb{R} \rightarrow \mathbb{R}$ is at least $C^{3}$ and satisfies

for some $\beta \geq \frac{1}{2}$.

$$
\left|G^{(k)}(t)\right| \leq C_{k}\langle t\rangle^{\beta-k},
$$

In 95 we prove a family of Strichartz-type estimates which will result in the following local well-posedness proposition. (See $₫ 5$ also for comments on optimality.)

Proposition 1.3. Suppose $(M, g)$ satisfies the assumptions of the introduction, $V \in \mathcal{C}_{c}^{\infty}(M)$, and in addition $M$ is asymptotically conic (as defined in [HTW]). Then for each

$$
s>\frac{n}{2}-\frac{k}{\max \{2 \beta-2,2\}}
$$

and each $u_{0} \in H_{D}^{s}(M)$ there exists $p>\max \{2 \beta-2,2\}$ and $0<T \leq 1$ such that (1.11) has a unique solution

$$
u \in C\left([-T, T] ; H_{D}^{s}(M)\right) \cap L^{p}\left([-T, T] ; L^{\infty}(M)\right) .
$$

Here $k=1$ if $\partial M \neq \emptyset$ and $k=2$ if $\partial M=\emptyset$. 
Moreover, the map $u_{0}(x) \mapsto u(t, x) \in C\left([-T, T] ; H_{D}^{s}(M)\right)$ is Lipschitz continuous on bounded sets of $H_{D}^{s}(M)$, and if $\left\|u_{0}\right\|_{H_{D}^{s}}$ is bounded, $T$ is bounded from below.

If we have $H^{1}$ energy conservation, Proposition 1.3 implies $u$ extends to a global solution.

Corollary 1.4. Suppose $(M, g)$ and $V$ satisfy the assumptions of Proposition 1.3. and assume $n \leq 2$. If $G(r) \rightarrow+\infty$ as $r \rightarrow+\infty$ then $u$ in (1.13) extends to a solution

$$
u \in C\left((-\infty, \infty) ; H_{D}^{1}(M)\right) \cap L^{p}\left((-\infty, \infty) ; L^{\infty}(M)\right) .
$$

If $\partial M=\emptyset, n \leq 3, \beta<3$, and $G(r) \rightarrow+\infty$ as $r \rightarrow+\infty$, then the same conclusion holds.

Remark 1.5. In particular, the cubic defocusing non-linear Schrödinger equation is globally well-posed. Observe also that three spatial dimensions is the smallest dimension in which the periodic orbit $\gamma$ can have a Poincaré map whose linearization possesses complex eigenvalues.

Local energy decay for the wave equation has also enjoyed a long history. Studied in non-trapping exterior domains by Morawetz [Mor, Morawetz-Phillips $\mathrm{MoPh}$, and Morawetz-Ralston-Strauss MRS, and generalized by, for example Vodev Vod, it is well-known (see Ral) that when there are trapped rays, exponential decay of the energy with no loss in derivative is impossible. Metcalfe-Sogge MeSo2 have recently shown that if there are trapped hyperbolic rays and exponential energy decay with loss in derivative, then one has long-time existence for certain classes of quasi-linear wave equations in $\mathbb{R}^{3}$. Theorem 2 says this always happens with one trapped hyperbolic orbit. Specifically, suppose $M=\mathbb{R}^{n} \backslash U$ for $U \Subset \mathbb{R}^{n},-\Delta$ is the Dirichlet Laplacian,

$$
Q(z, w) \in \mathcal{C}^{\infty}\left(\mathbb{C}^{n} \times \mathbb{C}^{n^{2}}\right)
$$

satisfies

i) $Q$ is linear in $w$,

ii) For each $w, Q(\cdot, w)$ is a symmetric quadratic form,

and consider the following initial value problem:

$$
\left\{\begin{array}{c}
\left(-D_{t}^{2}-\Delta\right) u=Q\left(D u, D^{2} u\right) \text { on } M \times[0, \infty), \\
u(x, 0)=u_{0}, D_{t} u(x, 0)=u_{1} .
\end{array}\right.
$$

The following Proposition then follows directly from [MeSo2, Theorem 1.1] in dimension $n=3$ and MeSo1, Theorem 1.1] in dimensions $n \geq 5$.

Proposition 1.6. Suppose $\left(u_{0}, u_{1}\right) \in\left(\mathcal{C}^{\infty}\left(\mathbb{R}^{n} \backslash U\right)\right)^{2}, n \geq 3$ odd, satisfy the compatibility condition from MeSo2, §1], and $\gamma \subset\left(\mathbb{R}^{n} \backslash U\right)$ is a trapped hyperbolic geodesic, with no other trapping. Assume further that if $n=3$, the null condition MeSo2, (1.9), (1.10)] holds. Then there exist $\epsilon_{0}>0$ and $N>0$ such that for every $\epsilon \leq \epsilon_{0}$, if

$$
\sum_{|\alpha| \leq N}\left\|\langle x\rangle^{|\alpha|} \partial_{x}^{\alpha} u_{0}\right\|_{L^{2}}+\sum_{|\alpha| \leq N-1}\left\|\langle x\rangle^{|\alpha|+1} \partial_{x}^{\alpha} u_{1}\right\|_{L^{2}} \leq \epsilon,
$$

then (1.14) has a unique solution $u \in \mathcal{C}^{\infty}\left([0, \infty) \times \mathbb{R}^{n} \backslash U\right)$. 
The manifolds we consider are the same as those studied (in the non-trapping case) in [CPV]. More precisely, let $(M, g)$ be a connected Riemannian manifold, $M=X_{0} \cup X$, where $X_{0}$ is a compact, connected $n$-dimensional Riemannian manifold and $X=\left[r_{0},+\infty\right) \times S, r_{0} \gg 1$, where $S$ is a compact, connected $(n-1)$ dimensional Riemannian manifold without boundary. We assume $\partial X_{0}$ is compact and that $X$ and $X_{0}$ satisfy

$$
\partial X_{0}=\partial M \cup \partial X, \partial M \cap \partial X=\emptyset .
$$

We assume the metric $\left.g\right|_{X_{0}}$ is a $\mathcal{C}^{\infty}$ metric on $\overline{X_{0}}$ and

$$
\left.g\right|_{X}=d r^{2}+\sigma(r)
$$

where $\sigma(r)$ is a family of smooth Riemannian metrics on $S$ depending smoothly on $r$. In local coordinates, the metric $\sigma(r)$ takes the form

$$
\sigma(r)=\sum_{i, j=1}^{n-1} g_{i j}(r, \theta) d \theta^{i} d \theta^{j}
$$

and if we set $X_{r}=[r,+\infty) \times S$, we can identify $\partial X_{r} \simeq(S, \sigma(r))$. Thus with $b=\left(\operatorname{det} g_{i j}\right)^{\frac{1}{2}}$ and $\left(g^{i j}\right)=\left(g_{i j}\right)^{-1}$, we have

$$
\Delta_{\partial X_{r}}=-b^{-1} \sum_{i, j} \partial_{\theta_{i}}\left(b g^{i j} \partial_{\theta_{j}}\right),
$$

and

$$
\Delta_{X}=-b^{-1} \partial_{r}\left(b \partial_{r}\right)+\Delta_{\partial X_{r}} .
$$

As in the introduction of [CPV], a calculation shows

$$
b^{\frac{1}{2}} \Delta_{X} b^{-\frac{1}{2}}=\partial_{r}^{2}+\Lambda_{r}+q(r, \theta),
$$

with

$$
\Lambda_{r}=-\sum_{i, j} \partial_{\theta_{i}}\left(g^{i j} \partial_{\theta_{j}}\right)
$$

and

$$
q(r, \theta)=(2 b)^{-2}\left(\frac{\partial b}{\partial r}\right)^{2}+(2 b)^{-2} \sum_{i, j} \frac{\partial b}{\partial \theta_{i}} \frac{\partial b}{\partial \theta_{j}} g^{i j}+\frac{1}{2} b \Delta_{X}\left(b^{-1}\right) .
$$

We assume $q(r, \theta)=q_{1}(r, \theta)+q_{2}(r, \theta)$, where

$$
\begin{aligned}
& \left|q_{1}(r, \theta)\right| \leq C, \quad\left|\frac{\partial^{k} q_{1}}{\partial r^{k}}\right| \leq C r^{-k-\delta} \text { for } k \geq 1, \text { and } \\
& \left|\frac{\partial^{k^{\prime}} q_{2}(r, \theta)}{\partial r^{k^{\prime}}}\right| \leq C r^{-k^{\prime}-1-\delta} \text { for } k^{\prime} \geq 0,
\end{aligned}
$$

for $C, \delta>0$. Observe this is satisfied for Euclidean space using a polar decomposition outside of a ball of radius $r_{0}$ (where $b=r^{n-1} \alpha(\theta)$ ), and for asymptotically Euclidean manifolds. Define $h \in \mathcal{C}^{\infty}\left(\left[r_{0},+\infty\right) \times T^{*}\left(\partial X_{r}\right)\right)$ by

$$
h(r, \theta, \xi)=\sum_{i, j} g^{i j}(r, \theta) \xi_{i} \xi_{j},
$$


and assume there is a constant $C>0$ such that for all $(\theta, \xi) \in T^{*}\left(\partial X_{r}\right)$,

$$
-\frac{\partial h}{\partial r}(r, \theta, \xi) \geq \frac{C}{r} h(r, \theta, \xi) .
$$

Let $-\Delta_{g}$ be the Laplace-Beltrami operator acting on functions, with Dirichlet boundary conditions if $\partial M \neq \emptyset, V \in \mathcal{C}^{\infty}(M), 0 \leq V \leq C$, satisfy (1.3), and let $P=-\Delta_{g}+V(x)$. The operator $P$ is an unbounded operator

$$
P: \mathcal{H} \rightarrow \mathcal{H},
$$

where $\mathcal{H}=L^{2}(M)$, with domain $H^{2}(M)$. In order to study the operator

$$
P-(\tau \pm i \epsilon),
$$

we use the following semiclassical rescaling for $-\Delta_{g}$. For $z \in[E-\delta, E+\delta]+$ $i\left(-c_{0} h, c_{0} h\right)$ and $\epsilon>0$ write

$$
\tau \pm i \epsilon=\frac{z}{h^{2}}
$$

Then

$$
\begin{aligned}
-\Delta_{g}+V(x)-(\tau \pm i \epsilon) & =-\Delta_{g}+V(x)-\frac{z}{h^{2}} \\
& =\frac{1}{h^{2}}\left(-h^{2} \Delta_{g}+h^{2} V(x)-z\right) .
\end{aligned}
$$

Now let $P(h)=-h^{2} \Delta_{g}+h^{2} V(x)$ be the self-adjoint semiclassical Schrödinger operator acting on $\mathcal{H}$ with Dirichlet boundary conditions if $\partial M \neq \emptyset$. Let $p=$ $\sigma_{h}(P(h)$ ) be the semiclassical (Weyl) principal symbol of $P(h)$ (see EvZw, Theorem D.3]). We assume the Hamiltonian flow of $H_{p}$ generates a single closed hyperbolic orbit $\gamma$ in the energy level $\{p=E\}, E>0$. Let $\pi: T^{*} M \rightarrow M$ denote the natural projection, and assume that the projected generalized geodesic $\pi(\gamma)$ lies entirely within $U_{0} \Subset U \Subset X_{0}$. If $\pi(\gamma)$ intersects $\partial M$, assume that the intersection is transversal. Assume further that the geometry is non-trapping outside $U_{0}$. That is, for every compact subset $K \Subset M \backslash U_{0}$, there is a time $T(K)$ so that if $\eta(t)$ is a generalized geodesic with $\eta(0) \in K$, there is a time $0<\tau \leq T(K)$ such that $\eta( \pm \tau) \in\left(M \backslash U_{0}\right) \backslash K$.

Acknowledgments. The author would like to thank Maciej Zworski for suggesting the local smoothing problem, as well as providing much help and support during the writing of this paper. He would also like to thank Jason Metcalfe for helpful comments and suggesting Proposition 1.6. Daniel Tataru for helpful discussions about optimality of Strichartz estimates, and Nicolas Burq for suggesting Proposition 5.9

\section{Resolvent estimates}

Let

$$
(P-(\tau \pm i \epsilon))^{-1}=\left(-\Delta_{g}+V(x)-(\tau \pm i \epsilon)\right)^{-1}
$$

be the classical resolvent. In this note we use the notation $\tau$ for the unsquared spectral parameter and $(\lambda \pm i \eta)^{2}=(\tau \pm i \epsilon)$ for the squared parameter. It will be convenient to use the lower half-plane as the physical half-plane. The proof of Theorem 1 relies on the weighted resolvent estimates of the following Theorem. 


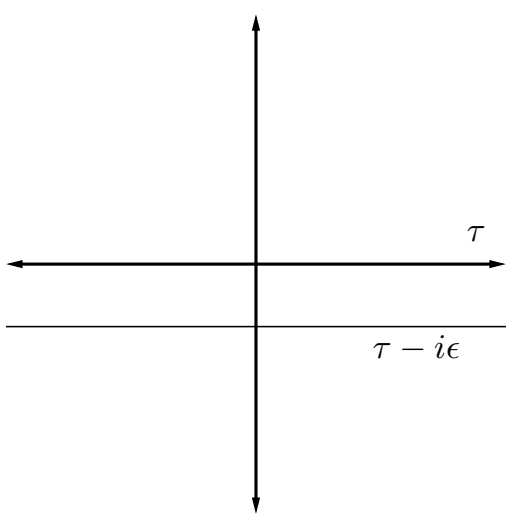

Figure 1. The curve $\tau-i \epsilon$ in the $z \in \mathbb{C}$ plane.

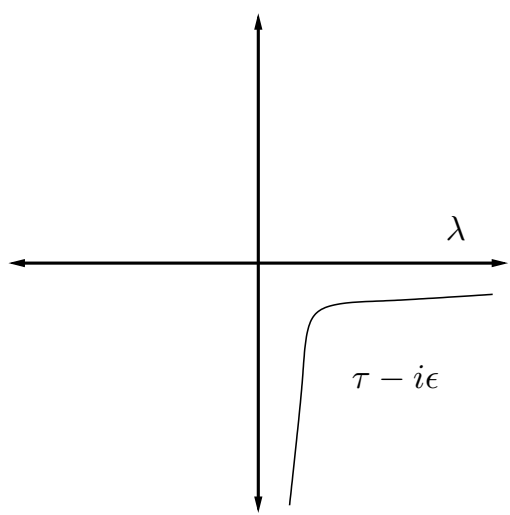

Figure 2. The same curve in the $z^{\frac{1}{2}}$ plane.

Theorem 3. Suppose $(M, g)$ satisfies all of the assumptions above. Then for each $\epsilon>0$ sufficiently small and each $s>\frac{1}{2}$ there is a constant $C>0$ such that

$$
\left\|\rho_{s}(P-(\tau \pm i \epsilon))^{-1} \rho_{s}\right\|_{\mathcal{H} \rightarrow \mathcal{H}} \leq C \frac{\log (2+|\tau|)}{\langle\tau\rangle^{1 / 2}} .
$$

Remark 2.1. To prove (2.1) is uniform in $\epsilon>0$, it suffices by Proposition 2.2 to prove the uniformity for $|\tau| \leq C$ for some $C>0$. This is the case if there are no embedded eigenvalues in $\mathbb{R}$. This is the case, for example, if $g$ is an asymptotically Euclidean scattering metric and $\partial M=\emptyset$, or if $(M, g)$ is equal to $\mathbb{R}^{n}$ outside a compact set. In the latter case, for $\psi$ satisfying (1.7),

$$
\psi\left(P-(\lambda \pm i \eta)^{2}\right)^{-1} \psi
$$

continues meromorphically to

$$
\lambda \pm i \eta \in\left\{\begin{array}{l}
\mathbb{C}, \quad n \text { odd, } \\
(\mathbb{C} \backslash\{0\})^{*}, \quad n \text { even, }
\end{array}\right.
$$

where $(\mathbb{C} \backslash\{0\})^{*}$ is the logarithmic Riemann surface. If, in addition, $V(x)$ satisfies (1.6), there is no pole at $\lambda=0$, and (2.1) is uniform in $\epsilon>0$ (see Vai, Theorem 8, Ch. 9]).

The contours we will be using are pictured in Figures 1 and 2 2 For details on the meromorphic continuation, see, for example, $\mathrm{Sjö}$.

To prove Theorem 3 in general, we observe

$$
\left\|\rho_{s}(P-(\tau \pm i \epsilon))^{-1} \rho_{s}\right\|_{\mathcal{H} \rightarrow \mathcal{H}} \leq \frac{C}{\epsilon} .
$$

Using this estimate for $|\tau| \leq C$, we need only show (2.1) for $|\tau|$ large, which is Corollary 2.3.

Choose $\chi \in \mathcal{C}_{c}^{\infty}(M), \chi \equiv 0$ near $\gamma$. We have

$$
\Delta_{g} \chi=\Delta_{0} \chi
$$

where $-\Delta_{0}$ is the Laplace-Beltrami operator associated to the nontrapping metric $\left.g\right|_{M \backslash U}$ on $M \backslash U$. Let $\chi_{s} \in \mathcal{C}^{\infty}(M), \chi_{s} \equiv 1$ on supp $\chi, \chi_{s} \equiv 0$ near $\gamma$, and 
$\chi_{s}(x) \equiv d_{g}\left(x, x_{0}\right)^{-s}$ for fixed $x_{0}$ and $x$ outside a compact set. Proposition 2.3 and the Remark immediately following from [CPV] show if $s>1 / 2$,

$$
\left\|\chi_{-s}(P(h)-E \pm i \epsilon) \chi u\right\|_{L^{2}(M)} \geq C h\left\|\chi_{s} u\right\|_{H_{h}^{1}(M)}
$$

for $h>0$ sufficiently small. Here $H_{h}^{1}(M)$ is the semiclassical Sobolev space equipped with the norm

$$
\|u\|_{H_{h}^{1}(V)}^{2}=\|u\|_{L^{2}(V)}^{2}+\|h \nabla u\|_{L^{2}(V)}^{2} .
$$

We prove the presence of $\gamma$ forces a weaker estimate.

Proposition 2.2. Let $(M, g)$ satisfy the assumptions of the introduction. For each $\rho_{s} \in \mathcal{C}^{\infty}(M)$ satisfying (1.2) there exist constants $C, h_{0}>0$ such that for $0<h \leq$ $h_{0}$

$$
\left\|\rho_{s}(P(h)-(E \pm i \epsilon))^{-1} \rho_{s}\right\|_{\mathcal{H} \rightarrow \mathcal{H}} \leq C h^{-1} \log (1 / h),
$$

uniformly in $\epsilon>0$.

From Proposition 2.2 we will be able to deduce the following Corollary by rescaling.

Corollary 2.3. Let $(M, g)$ satisfy the assumptions of the introduction. For each $\rho_{s} \in \mathcal{C}^{\infty}(M)$ satisfying (1.2), there exists a constant $C$ such that

$$
\left\|\rho_{s}\left(-\Delta_{g}+V(x)-(\tau \pm i \epsilon)\right)^{-1} \rho_{s}\right\|_{\mathcal{H} \rightarrow \mathcal{H}} \leq C \frac{\log (2+|\tau|)}{\langle\tau\rangle^{1 / 2}},
$$

for $|\tau| \geq C$ and $0<\epsilon \leq C^{-1}$.

Proof of Proposition 2.2. Observe for $\epsilon \geq c_{0} h / \log (1 / h)$, (2.4) holds automatically so we need only prove the Proposition for $\epsilon \leq c_{0} h / \log (1 / h)$ for some small constant $c_{0}>0$. Let $z \in[E-\delta, E+\delta]-i\left(c_{0} h / \log (1 / h), 0\right), \delta>0$, for the remainder of the proof.

The idea of the proof will be to glue two cutoff resolvent estimates together and control the interaction terms by propagation of singularities, and then replace the cutoffs with $\rho_{s}$, again controlling the erros with propagation of singularities. There are 4 main steps.

\section{Step 1: Select cutoffs.}

Fix $\psi \in \mathcal{C}_{c}^{\infty}(M), \psi \equiv 1$ in a neighbourhood of $\gamma$. Select $\chi, \chi_{1} \in \mathcal{C}_{c}^{\infty}(M), \chi \equiv 1$ near $\gamma$ with small support, $\chi_{1}=\psi-\chi$. Choose also $\chi_{s}, s>\frac{1}{2}$, satisfying (1.2) with $\chi_{s} \equiv 1$ on $\operatorname{supp} \chi_{1}$ and $\chi_{s} \equiv 0$ near $\gamma$.

In order to control the interaction (commutator) terms, we will add a complex absorption potential to $P(h)-z$ which is supported away from the above cutoffs, which will control the interactions through propagation of singularities. Recall we have defined $X_{r}=[r,+\infty) \times S$ in the introduction. Assume supp $\psi \subset M \backslash X_{R_{0}}$, $R_{0}>0$. Choose $R_{j}, j=1, \ldots, 7$,

$$
R_{0}<R_{1}, \quad R_{j}<R_{j+1}<\infty,
$$

and let

$$
A_{R_{j_{1}}, R_{j_{2}}}=X_{R_{j_{1}}} \backslash X_{R_{j_{2}}}
$$

be the annulus with inner radius $R_{j_{1}}$ and outer radius $R_{j_{2}}$. 
Choose $a \in \mathcal{C}_{c}^{\infty}(M), a \psi \equiv 0$,

$$
a \equiv 1 \text { on } A_{R_{1}, R_{6}},
$$

and choose $\psi_{1}, \psi_{2} \in \mathcal{C}_{c}^{\infty}(M)$ satisfying

$$
\begin{aligned}
& \operatorname{supp} \psi_{1} \subset A_{R_{1}, R_{6}}, \psi_{1} \equiv 1 \text { on } A_{R_{2}, R_{5}}, \\
& \operatorname{supp} \psi_{2} \subset A_{R_{2}, R_{5}}, \psi_{2} \equiv 1 \text { on } A_{R_{3}, R_{4}} .
\end{aligned}
$$

Set $Q(z)=P(h)-z-i C_{1} h a$ for a constant $C_{1}>0$ to be chosen later in the proof. Recall

$$
\gamma \Subset U_{0} \Subset U \Subset X_{0},
$$

and choose $\tilde{\chi} \in \mathcal{C}_{c}^{\infty}(M)$ satisfying

$$
\begin{aligned}
\tilde{\chi} & \equiv 1 \text { on } M \backslash X_{R_{6}} \backslash U \text { and } \\
\operatorname{supp} \tilde{\chi} & \subset M \backslash X_{R_{7}} \backslash U_{0} .
\end{aligned}
$$

Without loss of generality, we assume $\rho_{s}$ from the statement of the Proposition satisfies (1.2) and $\rho_{s} \equiv 1$ on $M \backslash X_{R_{7}}$

Choose $\chi_{2} \in \mathcal{C}_{c}^{\infty}(M), \chi_{3} \in \mathcal{C}^{\infty}(M)$, such that $\chi_{2}+\chi_{3} \equiv 1$,

$$
\chi_{2} \equiv 1 \text { near } \gamma, \chi_{3} \equiv 0 \text { near } \gamma \text {, }
$$

and

$$
\left|\nabla \chi_{j}\right| \leq \frac{\beta}{E+\alpha} \ll 1
$$

for $0<\delta<\alpha$. Choose also $\tilde{\chi}_{2} \in \mathcal{C}_{c}^{\infty}(M), \tilde{\chi}_{3} \in \mathcal{C}^{\infty}(M), \tilde{\chi}_{j} \equiv 1$ on $\operatorname{supp} \chi_{j}$, and $\left|\nabla \tilde{\chi}_{j}\right| \leq \beta /(E+\alpha)$. Here $\beta>0$ will be fixed later in the proof, and we assume $R_{0}$ is chosen sufficiently large that supp $\chi_{j} \subset M \backslash X_{R_{0}}$ for $j=2,3$.

These cutoffs are shown in Figure 3 .

We will also employ an energy cutoff, to separate the characteristic variety of $p-E$ from the elliptic sets. Choose $\varphi_{1} \in \mathcal{C}_{c}^{\infty}(\mathbb{R})$,

$$
\varphi_{1}(t) \equiv 1 \text { on }\{|t| \leq \alpha / 2\}, \varphi_{1}(t) \equiv 0 \text { on }\{|t| \geq \alpha\} \text {. }
$$

Set

$$
\varphi(x, \xi)=\varphi_{1}(p(x, \xi)-E),
$$

and observe since $\varphi$ is a function of the principal symbol of $P(h)-z$ and we are using the Weyl calculus,

$$
\left[P(h)-z, \varphi^{w}\right]=\mathcal{O}\left(h^{3}\right)
$$

\section{Step 2: Microlocalization.}

We will bound $\|\psi u\|$ from above, where unless explicitly noted, $\|\cdot\|=\|\cdot\|_{\mathcal{H}}$. To do this, calculate

$$
\|\psi u\|=\|\psi u\| \leq\left\|\chi_{1} u\right\|+\|\chi u\|=: A+B .
$$

Using [CPV, Theorem 1.1], we have

$$
\begin{aligned}
A & \leq C h^{-1}\left\|\rho_{-s}(P(h)-z) \chi_{1} u\right\| \\
& \leq C h^{-1}\left(\left\|\rho_{-s} \chi_{1}(P(h)-z) u\right\|+\left\|\rho_{-s}\left[P(h), \chi_{1}\right] u\right\|\right) \\
& \leq C h^{-1}\left(\|Q(z) u\|+\left\|\left[P(h), \chi_{1}\right] u\right\|\right),
\end{aligned}
$$

since $\chi_{1} a=0$ and $\rho_{-s}$ is bounded on the support of $\chi_{1}$. 

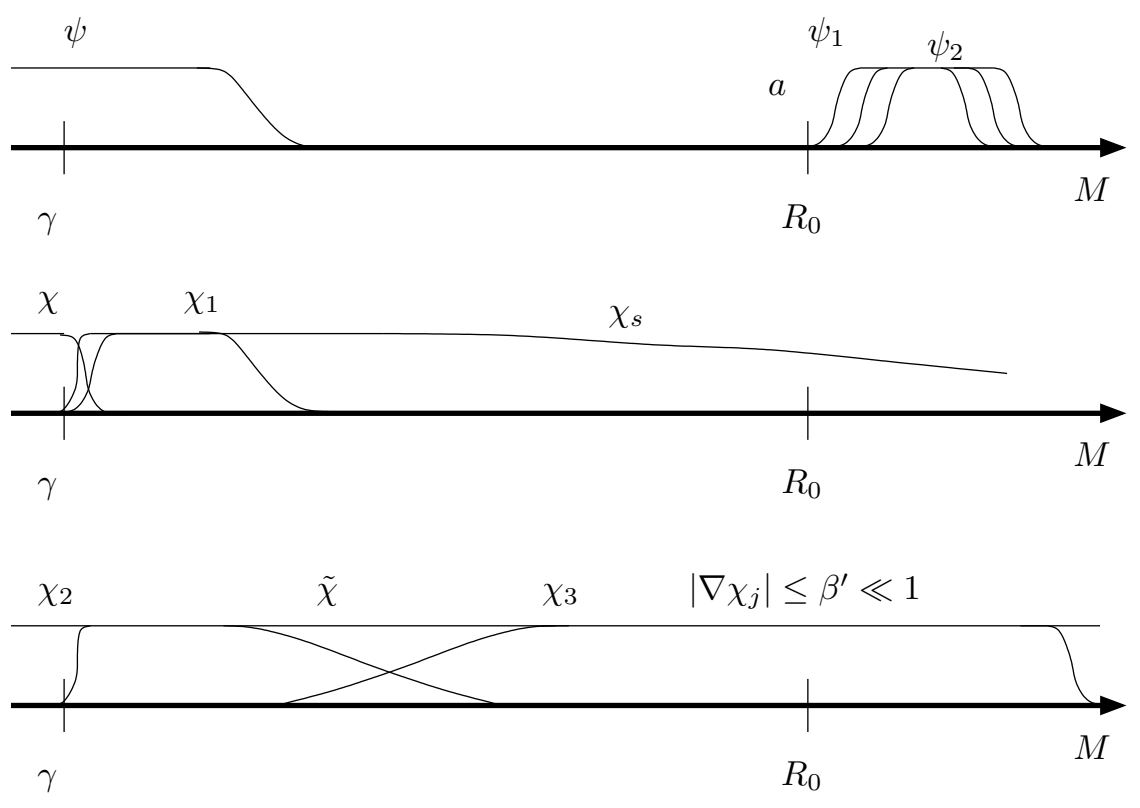

Figure 3. The manifold $M$ with various cutoff functions employed in the proof of Proposition 2.2. Here $\beta^{\prime}=\beta /|E+\alpha|$.

For $B$ we cutoff also in the energy to apply [Chr1, Theorem 1, Corollary 8] and the generalizations from Chr2 for $c_{0}>0$ sufficiently small:

$$
\begin{aligned}
B \leq & \left\|\varphi^{w} \chi u\right\|+\left\|(1-\varphi)^{w} \chi u\right\| \\
\leq & \frac{C \log (1 / h)}{h}\left\|(P(h)-z) \varphi^{w} \chi u\right\|+\frac{C}{\alpha}\left\|(P(h)-z)(1-\varphi)^{w} \chi u\right\| \\
\leq & \frac{C \log (1 / h)}{h}\left\|\varphi^{w}(P(h)-z) \chi u\right\|+C h^{2} \log (1 / h)\|\chi u\| \\
& +\frac{C}{\alpha}\left\|(1-\varphi)^{w}(P(h)-z) \chi u\right\|+\frac{C}{\alpha} h^{3}\|\chi u\| \\
\leq & \frac{C \log (1 / h)}{h}(\|Q(z) u\|+\|[P(h), \chi] u\|)+C h^{2} \log (1 / h)\|\chi u\|,
\end{aligned}
$$

since $\chi a=0$. Here in (2.6 2.7) we have used (2.5).

\section{Step 3: Two Lemmas.}

The first Lemma is a refinement of the standard propagation of singularities result.

Lemma 2.4. Let $V_{1}, V_{2} \Subset T^{*} M$, and let $\widetilde{V}_{1}, \widetilde{V}_{2} \Subset M$ be the $x$-projections of $V_{1}$ and $V_{2}$ respectively. Suppose the $\widetilde{V}_{j}$ satisfy dist $g\left(\widetilde{V}_{1}, \widetilde{V}_{2}\right)=\frac{T}{K}$ for some $K>0$, and

$$
\left\{\begin{array}{l}
\forall \rho \text { in a neighbourhood of } V_{1}, \quad \exists 0<t<T \text { and } \epsilon= \pm 1 \\
\text { such that } \exp \left(\epsilon t H_{p}\right)(\rho) \in V_{2} .
\end{array}\right.
$$

Suppose $A \in \Psi_{h}^{0,0}$ is microlocally equal to 1 in $V_{2}$. If $B \in \Psi_{h}^{0,0}$ and $\mathrm{WF}_{h}(B) \subset V_{1}$, then

$$
\|B u\| \leq C T h^{-1}\|P(h) u\|+2 e^{2} K\|B\|_{\mathcal{H} \rightarrow \mathcal{H}}\|A u\|+\mathcal{O}(h)\|\widetilde{B} u\|,
$$


where

$$
\widetilde{B} \equiv 1 \text { on } \cup_{0 \leq t \leq T} \exp \left(\epsilon t H_{p}\right)\left(\mathrm{WF}_{h} B\right) .
$$

Remark 2.5. Observe Lemma 2.4 is a statement about the principal symbol $p-z$, and hence applies also to $Q(z)$, and the difference is $\mathcal{O}\left(h^{\infty}\right)\|\widetilde{B} u\|$.

Proof. This lemma is well known except for the constant $2 e^{2} K\|B\|_{\mathcal{H} \rightarrow \mathcal{H}}$ in front of the control term. We therefore only sketch the proof taking note of where this constant arises. Let $G=\mathrm{Op}^{w}(g) \in \Psi_{h}^{0,0}$ be a self-adjoint operator to be determined later in the proof and calculate

$$
\begin{aligned}
\frac{T}{2 h}\|G(P-z) u\|^{2}+\frac{h}{2 T}\|G u\|^{2} & \geq \operatorname{Im}\langle G(P-z) u, G u\rangle \\
& =\operatorname{Im}\langle[G, P] u, G u\rangle \\
& \geq \frac{h}{2}\left\langle\operatorname{Op}^{w}\left(\left\{p, g^{2}\right\}\right) u, u\right\rangle-\mathcal{O}\left(h^{3}\right)\|\widetilde{G} u\|^{2},
\end{aligned}
$$

where $\widetilde{G} \equiv 1$ on $\mathrm{WF}_{h} G$. Consequently, we consider the symbol

$$
\alpha(x, \xi)=\left\{p, g^{2}\right\}-g^{2} / T .
$$

Choose $\varphi_{0} \in \mathcal{C}^{\infty}(M), 0 \leq \varphi_{0} \leq 1$, satisfying

$$
\begin{aligned}
\varphi_{0} & =\varphi_{1}^{2}, \text { for } \varphi_{1} \in \mathcal{C}^{\infty}(M), \\
\varphi_{0} & \equiv 1 \text { on } \widetilde{V}_{1}, \\
\varphi_{0} & \equiv 0 \text { on } \widetilde{V}_{2}, \text { and } \\
\left|\nabla \varphi_{0}\right| & \leq \frac{2 K}{T} .
\end{aligned}
$$

Choose also $\varphi \in \mathcal{C}^{\infty}\left(T^{*} M\right), \varphi=\varphi(p(x, \xi)-E)$ so that $\varphi^{2} \varphi_{0} \equiv 1$ on $V_{1}$, and $\operatorname{supp} \varphi^{2} \varphi_{0}$ satisfies (2.8). From [EvZw, Theorem 10.18], we know that in a sufficiently small neighbourhood near $\{p=E\}$ there are symplectic coordinates so that $p=\xi_{1}$. Thus in local coordinates we choose

$$
g^{2}=\varphi^{2}(p-E) \varphi_{0}(x) \exp \left(2 x_{1} / T\right),
$$

so that we have the pointwise estimate

$$
|\alpha(x, \xi)| \geq \frac{1}{T} \varphi^{2} \varphi_{0}-\varphi^{2}\left|\nabla \varphi_{0}\right| e^{2} .
$$

Combining (2.9) with (2.10) gives the lemma.

The second Lemma will follow from Lemma 2.4 and indicates how to control the interaction terms of the form $\|[P, \chi] u\|$.

Lemma 2.6. Suppose $\chi \in \mathcal{C}_{c}^{\infty}(M)$ satisfies

$$
\operatorname{supp} \chi \subset M \backslash X_{R_{0}} \text {, and } \nabla \chi \equiv 0 \text { near } \gamma \text {. }
$$

Then

$$
h^{-1}\|[P(h), \chi] u\| \leq C R_{0} h^{-1}\|Q(z) u\|+\mathcal{O}(h)\|\tilde{\chi} u\|,
$$

where $\tilde{\chi} \in \mathcal{C}_{c}^{\infty}(M), \tilde{\chi} \equiv 0$ on a neighbourhood of $\gamma$, and

$$
\tilde{\chi} \equiv 1 \text { on } M \backslash X_{R_{6}} \backslash U,
$$

where $U \supset \gamma$ is a small neighbourhood. 
Proof. We first microlocalize using $\varphi^{w}$ as in Step 2. Observe $[P(h), \chi]=h A(x, h D)$, where $A(x, h D)$ is a first order semiclassical differential operator with coefficients supported in $M \backslash X_{R_{0}} \backslash \operatorname{neigh}(\gamma)$. We calculate

$$
\|A(x, h D) u\| \leq\left\|A(x, h D) \varphi^{w} u\right\|+\left\|A(x, h D)(1-\varphi)^{w} u\right\| .
$$

Now

$$
\left\|A(x, h D) \varphi^{w} u\right\| \leq|E+\alpha|\|\nabla \chi\|_{L^{\infty}}\left\|\varphi_{2}^{w} u\right\|
$$

for $\varphi_{2} \in \mathcal{C}_{c}^{\infty}\left(T^{*} M\right), 0 \leq \varphi_{2} \leq 1$, a microlocal cutoff supported away from $\gamma$. From Lemma 2.4 we have

$$
\begin{aligned}
& |E+\alpha|\|\nabla \chi\|_{L^{\infty}}\left\|\varphi_{2}^{w} u\right\| \\
& \quad \leq C R_{0} h^{-1}\|Q(z) u\|+C\|\nabla \chi\|_{L^{\infty}}\left\|\psi_{1} u\right\|+\mathcal{O}(h)\|\tilde{\chi} u\| .
\end{aligned}
$$

For the second term in (2.11) choose $\tilde{\chi}_{2} \in \mathcal{C}_{c}^{\infty}(M)$ satisfying $\tilde{\chi}_{2} \equiv 1$ on $\operatorname{supp} \nabla \chi$ with support in a slightly larger set. We calculate:

$$
\begin{aligned}
\left\|A(x, h D)(1-\varphi)^{w} u\right\|^{2} & =\left\langle A(x, h D)^{*} A(x, h D)(1-\varphi)^{w} u, \tilde{\chi}(1-\varphi)^{w} u\right\rangle \\
& \leq \frac{1}{2}\left\|A^{*} A(1-\varphi)^{w} u\right\|^{2}+\frac{1}{2}\left\|\tilde{\chi}_{2}(1-\varphi)^{w} u\right\|^{2} \\
& \leq \frac{C}{\alpha}\left\|(P(h)-z) \tilde{\chi}_{2}(1-\varphi)^{w} u\right\|^{2} \\
& \leq \frac{C}{\alpha}\|Q(z) u\|^{2}+C h^{2}\|\nabla \chi\|_{L^{\infty}}\left\|\psi_{1} u\right\|^{2}+\mathcal{O}\left(h^{4}\right)\|\tilde{\chi} u\|^{2},
\end{aligned}
$$

where we have again used Lemma 2.4. (2.5) and the fact that $P(h)-z$ is a second order elliptic semiclassical differential operator on $\operatorname{supp}(1-\varphi)^{w}$.

We have shown

$$
h^{-1}\|[P(h), \chi] u\| \leq C R_{0} h^{-1}\|Q(z) u\|+C\|\nabla \chi\|_{L^{\infty}}\left\|\psi_{1} u\right\|+\mathcal{O}(h)\|\tilde{\chi} u\| .
$$

Now choose $C_{1}>0$ sufficiently large that

$$
\left(C_{1} a-c_{0} / \log (1 / h)\right) \psi_{1} \geq\left(C_{1} a-c_{0}\right) \psi_{1} \geq\left\{\begin{array}{l}
c_{0} \psi_{1} / 2 \\
c_{0} \psi_{2}^{2}
\end{array}\right.
$$

Then

$$
\begin{aligned}
\frac{1}{2} c_{0} h \int\left|\psi_{2} u\right|^{2} d x & \leq h \int\left(C_{1} a+\operatorname{Im} z / h\right) u \overline{\psi_{1} u} d x \\
& \leq-\operatorname{Im} \int Q(z) u \overline{\psi_{1} u} d x \\
& \leq(4 \eta h)^{-1}\|Q(z) u\|^{2}+\eta h\left\|\psi_{1} u\right\|^{2} .
\end{aligned}
$$

Now Lemma 2.4 gives with $B=\psi_{1}$ and $A=\psi_{2}$,

$$
\left\|\psi_{1} u\right\| \leq C h^{-1}\|Q(z) u\|+C\left\|\psi_{2} u\right\|+\mathcal{O}(h)\|\tilde{\chi} u\| .
$$

Combining the last two inequalities yields

$$
\begin{aligned}
\left\|\psi_{1} u\right\|^{2} & \leq C^{2} h^{-2}\|Q(z) u\|^{2}+C h^{-1}\|Q(z) u\|\left\|\psi_{2} u\right\|+\left\|\psi_{2} u\right\|^{2}+\mathcal{O}\left(h^{2}\right)\|\tilde{\chi} u\|^{2} \\
& \leq C^{2} h^{-2}\|Q(z) u\|^{2}+\eta\left\|\psi_{1} u\right\|^{2}+\mathcal{O}\left(h^{2}\right)\|\tilde{\chi} u\| .
\end{aligned}
$$

For sufficiently small $\eta>0$ independent of $h$, this yields

$$
h^{-1}\|[P(h), \chi] u\| \leq C h^{-1} R_{0}\|Q(z) u\|+\mathcal{O}(h)\|\tilde{\chi} u\| .
$$


Step 4: $(P-z)^{-1}$ and $\rho_{s}$.

We have shown

$$
\|\psi u\| \leq \frac{C \log (1 / h)}{h} R_{0}\|Q(z) u\|+\mathcal{O}(h)\|\tilde{\chi} u\|,
$$

but we have yet to replace $Q(z)$ in the estimate with $P(h)-z$ and add the weights $\rho_{s}$. Equation (2.12) implies

$$
\begin{aligned}
\left\|\tilde{\chi}_{2} u\right\| & =\left\|\tilde{\chi}_{2} \chi_{2} u\right\| \\
& \leq \frac{C \log (1 / h)}{h}\left\|Q(z) \chi_{2} u\right\|+\mathcal{O}(h)\left\|\chi_{2} u\right\| \\
& \leq \frac{C \log (1 / h)}{h}\left\|(P(h)-z) \chi_{2} u\right\|+\mathcal{O}(h)\left\|\chi_{2} u\right\|
\end{aligned}
$$

since $a \chi_{2}=0$. The last term on the right hand side is controlled by the left hand side, so

$$
\left\|\tilde{\chi}_{2} u\right\| \leq \frac{C^{\prime} \log (1 / h)}{h}\left\|(P(h)-z) \chi_{2} u\right\| .
$$

Applying again [CPV, Theorem 1.1], we get

$$
\begin{aligned}
\left\|\rho_{s} \tilde{\chi}_{3} u\right\| & =\left\|\rho_{s} \tilde{\chi}_{3} \chi_{3} u\right\| \\
& \leq C h^{-1}\left\|\rho_{-s} \tilde{\chi}_{3}(P(h)-z) \chi_{3} u\right\| .
\end{aligned}
$$

Thus

$$
\begin{aligned}
& C\left\|\rho_{-s}(P(h)-z) u\right\|^{2} \\
& \geq C_{2}\left(\left\|\rho_{-s} \chi_{3}(P(h)-z) u\right\|^{2}+\left\|\chi_{2}(P(h)-z) u\right\|^{2}\right) \\
& \geq \quad\left\|\rho_{-s}(P(h)-z) \chi_{3} u\right\|^{2}+\left\|(P(h)-z) \chi_{2} u\right\|^{2} \\
&+\left\|\left[P(h), \chi_{3}\right] \tilde{\chi}_{3} u\right\|^{2}+\left\|\left[P(h), \chi_{2}\right] \tilde{\chi}_{2} u\right\|^{2} \\
& \quad-2 \sum_{j=2}^{3}\left\|\left(\rho_{-s}\right)^{j-2}(P(h)-z) \chi_{j} u\right\|\left\|\left[P(h), \chi_{j}\right] \tilde{\chi}_{j} u .\right\|
\end{aligned}
$$

Applying the Cauchy-Schwarz inequality to the last term on the right hand side yields

$$
\begin{aligned}
& \left\|\left(\rho_{-s}\right)^{j-2}(P(h)-z) \chi_{j} u\right\|\left\|\left[P(h), \chi_{j}\right] \tilde{\chi}_{j} u\right\| \\
& \quad \leq\left(\left\|\left(\rho_{-s}\right)^{j-2} \chi_{j}(P(h)-z) u\right\|+\left\|\left[P(h), \chi_{j}\right] \tilde{\chi}_{j} u\right\|\right)\left\|\left[P(h), \chi_{j}\right] \tilde{\chi}_{j} u\right\| \\
& \quad \leq \frac{1}{2}\left\|\left(\rho_{-s}\right)^{j-2} \chi_{j}(P(h)-z) u\right\|^{2}+\frac{3}{2}\left\|\left[P(h), \chi_{j}\right] \tilde{\chi}_{j} u\right\|^{2} .
\end{aligned}
$$

Plugging into (2.13), we have

$$
\begin{aligned}
C_{3}\|(P(h)-z) u\|^{2} \geq & \left\|\rho_{-s}(P(h)-z) \chi_{3} u\right\|^{2}+\left\|(P(h)-z) \chi_{2} u\right\|^{2} \\
& -2\left\|\left[P(h), \chi_{3}\right] \tilde{\chi}_{3} u\right\|^{2}-2\left\|\left[P(h), \chi_{2}\right] \tilde{\chi}_{2} u\right\|^{2} .
\end{aligned}
$$

Applying Lemma 2.4 to the last two terms in (2.15) with $A=\rho_{s} \tilde{\chi}_{3}$, we get

$$
\left\|\left[P(h), \chi_{j}\right] \tilde{\chi}_{j} u\right\| \leq C^{\prime} R_{0}\|(P(h)-z) u\|+C \beta h\left\|\rho_{s} \tilde{\chi}_{3} u\right\|+\mathcal{O}\left(h^{2}\right)\|\tilde{\chi} u\|,
$$


with $C$ here independent of $\beta$ and $h$. Hence

$$
\begin{aligned}
C_{3}\left\|\rho_{-s}(P(h)-z) u\right\|^{2} \geq & \left\|\rho_{-s}(P(h)-z) \chi_{3} u\right\|^{2}+\left\|(P(h)-z) \chi_{2} u\right\|^{2} \\
& -4 C \beta^{2} h^{2}\left\|\rho_{s} \tilde{\chi}_{3} u\right\|^{2}-\mathcal{O}\left(h^{4}\right)\|\tilde{\chi} u\|^{2} \\
\geq & \frac{h^{2}}{C^{2}}\left\|\rho_{s} \tilde{\chi}_{3} u\right\|^{2}+\frac{h^{2}}{C^{2} \log ^{2}(1 / h)}\left\|\tilde{\chi}_{2} u\right\| \\
& -4 C \beta^{2} h^{2}\left\|\rho_{s} \tilde{\chi}_{3} u\right\|^{2}-\mathcal{O}\left(h^{4}\right)\|\tilde{\chi} u\|^{2} \\
\geq & \frac{h^{2}}{C_{4} \log ^{2}(1 / h)}\left\|\rho_{s} u\right\|^{2},
\end{aligned}
$$

as long as $\beta>0$ is sufficiently small. This is (2.4).

Theorem 3 now follows immediately from Corollary 2.3 ,

\section{Proof of Theorem 1}

In this section we show how to use the estimate (2.4) to prove Theorem 1. This is an adaptation of the similar proof in Bur2, in the case $M$ is Euclidean space with several convex bodies removed and compactly supported weights.

Let $\rho_{s}$ satisfy (1.2), let $\mu=\tau \pm i \epsilon$, and suppose $u$ and $f$ satisfy

$$
\left(\Delta_{g}-V+\mu\right) u=\rho_{s} f .
$$

We multiply by $\rho_{s}^{2} \bar{u}$ and integrate:

$$
\begin{aligned}
& \int \rho_{s}^{2} \bar{u} \Delta u+\int(\mu-V) \rho_{s}^{2}|u|^{2}=\int \rho_{s}^{3} f \bar{u} \\
& \Longrightarrow-\int \rho_{s}^{2}|\nabla u|^{2}+\int \mu \rho_{s}^{2}|u|^{2}-\int\left(\nabla u, \nabla\left(\rho_{s}^{2}\right)\right) \bar{u}=\int \rho_{s}^{3} f \bar{u}
\end{aligned}
$$

which implies

$$
\begin{aligned}
\int \rho_{s}^{2}|\nabla u|^{2} \leq(|\tau| & +C) \int \rho_{s}^{2}|u|^{2}+\beta \int|\nabla u|^{2}\left|\nabla\left(\rho_{s}^{2}\right)\right|^{2} \rho_{-s}^{2} \\
& +(4 \beta)^{-1} \int \rho_{s}^{2}|u|^{2}+\left|\int \rho_{s}^{3} f \bar{u}\right|
\end{aligned}
$$

for any $\beta>0$, since $V$ is bounded. We observe

$$
\left|\nabla\left(\rho_{s}^{2}\right)\right| \leq C\langle x\rangle^{-2 s-1}
$$

for large $|x|$, and hence

$$
\left|\nabla\left(\rho_{s}^{2}\right)\right|^{2} \rho_{-s}^{2} \leq C \rho_{s}^{2}
$$

for large $|x|$. This combined with $\rho_{s}^{2} \leq C \rho_{s}$ implies

$$
\int \rho_{s}^{2}|\nabla u|^{2} \leq(|\tau|+C)\left\|\rho_{s} u\right\|_{L^{2}}^{2}+\left\|\rho_{s} f\right\|_{L^{2}}^{2} .
$$

Now (3.1) implies

$$
\begin{aligned}
(|\tau|+C)^{1 / 2}\left\|\rho_{s} u\right\|_{L^{2}} & \leq(|\tau|+C)^{1 / 2}\left\|\rho_{s}\left(\Delta_{g}-V+\mu\right)^{-1} \rho_{s} f\right\|_{L^{2}} \\
& \leq C \log (2+|\tau|)\|f\|_{L^{2}}
\end{aligned}
$$


which combined with (3.1) gives

$$
\left\|\rho_{s} u\right\|_{H^{1}}^{2} \leq C \int \rho_{s}^{2}|\nabla u|^{2}+C \int\left\|\rho_{s} u\right\|_{L^{2}}^{2} \leq C \log (2+|\tau|)\|f\|_{L^{2}}^{2} .
$$

This combined with the standard interpolation arguments gives the following lemma.

Lemma 3.1. With the notation and assumptions above, we have

$$
\left\|\rho_{s}\left(-\Delta_{g}+V-(\tau \pm i \epsilon)\right)^{-1} \rho_{s}\right\|_{L^{2} \rightarrow H^{1}} \leq C_{\epsilon} \log (2+|\tau|)
$$

and for every $\delta>0, r \in[-1,1]$,

$$
\left\|\rho_{s}\left(-\Delta_{g}+V-(\tau \pm i \epsilon)\right)^{-1} \rho_{s}\right\|_{H^{r} \rightarrow H^{1+r-\delta}} \leq C_{\epsilon, \delta} .
$$

Now let $A$ be the operator

$$
A u_{0}=\rho_{s} e^{-i t P} u_{0},
$$

acting on $L^{2}(M)$. We want to show

$$
A: L^{2}(M) \rightarrow L^{2}\left([0, T] ; H^{\frac{1}{2}-\epsilon}(M)\right)
$$

is bounded. We use the standard argument from BGT2. That is, by duality, this is equivalent to the adjoint $A^{*}$ being bounded

$$
A^{*}: L^{2}\left([0, T] ; H^{-\frac{1}{2}+\epsilon}(M)\right) \rightarrow L^{2}(M)
$$

which is equivalent to the boundedness of

$$
A A^{*}: L^{2}\left([0, T] ; H^{-\frac{1}{2}+\epsilon}(M)\right) \rightarrow L^{2}\left([0, T] ; H^{\frac{1}{2}-\epsilon}(M)\right) .
$$

The definition of $A$ gives

$$
A^{*} f=\int_{0}^{T} e^{i \tau P} \rho_{s} f(\tau) d \tau
$$

so

$$
A A^{*} f(t)=\int_{0}^{T} \rho_{s} e^{-i(t-\tau) P} \rho_{s} f(\tau) d \tau .
$$

We show $A A^{*}$ is bounded. Let $u$ be defined by

$$
u(x, t)=\int_{0}^{T} e^{-i(t-\tau) P} \rho_{s} f(\tau) d \tau .
$$

Since we are only interested in the time interval $[0, T]$, we extend $f$ to be 0 for $t \notin[0, T]$. We write

$$
\begin{aligned}
A A^{*} f(t) & =\int_{0}^{t} \rho_{s} e^{-i(t-\tau) P} \rho_{s} f(\tau) d \tau+\int_{t}^{T} \rho_{s} e^{-i(t-\tau) P} \rho_{s} f(\tau) d \tau \\
& =: \quad \rho_{s} u_{1}(t)+\rho_{s} u_{2}(t),
\end{aligned}
$$

and calculate

$$
\left(D_{t}+P\right) u_{j}=(-1)^{j} i \rho_{s} f .
$$

Thus boundedness of $A A^{*}$ will follow if we prove $u$ satisfying (3.3) satisfies

$$
\left\|\rho_{s} u\right\|_{L^{2}\left([0, T] ; H^{\frac{1}{2}-\epsilon}\right)} \leq\|f\|_{L^{2}\left([0, T] ; H^{-\frac{1}{2}+\epsilon}\right)} .
$$


Replacing \pm if with $f$ in equation (3.3) and taking the Fourier transform in time results in the following equation for $\hat{u}$ and $\hat{f}$ :

$$
(-z+P) \hat{u}(z, \cdot)=\rho_{s} \hat{f}(z, \cdot) .
$$

Since $f(t, \cdot)$ is supported only in $[0, T], \hat{f}(z, \cdot)$ and $\hat{u}(z, \cdot)$ are holomorphic, bounded, and satisfy (3.4) in $\{\operatorname{Im} z<0\}$. Let $z=\tau-i \eta, \eta>0$ sufficiently small. We apply Lemma 3.1 to get

$$
\left\|\rho_{s} \hat{u}(z, \cdot)\right\|_{H^{\frac{1}{2}-\epsilon}(M)} \leq C\|\hat{f}(z, \cdot)\|_{H^{-\frac{1}{2}+\epsilon}(M)},
$$

for $\epsilon>0$. Thus

$$
\begin{aligned}
\left\|\rho_{s} u\right\|_{L^{2}\left([0, T] ; H^{\frac{1}{2}-\epsilon}(M)\right)} & \leq e^{\eta T}\left\|e^{-\eta t} \rho_{s} u(t)\right\|_{L^{2}\left([0, T] ; H^{\frac{1}{2}-\epsilon}(M)\right)} \\
& \leq C e^{\eta T}\left\|\rho_{s} \hat{u}(\tau-i \eta)\right\|_{L^{2}\left(\mathbb{R} ; H^{\frac{1}{2}-\epsilon}(M)\right)} \\
& \leq C e^{\eta T}\|\hat{f}(\tau-i \eta)\|_{L^{2}\left(\mathbb{R} ; H^{-\frac{1}{2}+\epsilon}(M)\right)} \\
& \leq C e^{\eta T}\left\|e^{-\eta t} f(t)\right\|_{L^{2}\left([0, T] ; H^{-\frac{1}{2}+\epsilon}(M)\right)} \\
& \leq C e^{\eta T}\|f(t)\|_{L^{2}\left([0, T] ; H^{-\frac{1}{2}+\epsilon}(M)\right)}
\end{aligned}
$$

Hence

$$
\int_{0}^{T}\left\|\rho_{s} u\right\|_{H^{\frac{1}{2}-\epsilon}(M)}^{2} d t \leq C e^{\eta T} \int_{0}^{T}\|f\|_{H^{-\frac{1}{2}+\epsilon}(M)}^{2} d t
$$

or $A A^{*}$ is bounded. Thus $A$ is bounded and Theorem 1 is proved.

Remark 3.2. If the estimate (2.1) is uniform in the lower half-plane, then the preceding calculation can be made including taking the limit $\eta \rightarrow 0$, in which case we get the global in time local smoothing estimate (1.4)

The following Lemma uses interpolation to replace the $H^{1 / 2-\epsilon}$ norm on the left hand side of (1.1) with $H^{1 / 2}$, and will be of use in 95 .

Lemma 3.3. Suppose $(M, g)$ and $V$ satisfy the assumptions of Theorem 1 . For each $\delta>0$ there is a constant $C>0$ such that

$$
\int_{0}^{T}\left\|\rho_{s} e^{i t\left(\Delta_{g}-V(x)\right)} u_{0}\right\|_{H^{1 / 2}(M)}^{2} d t \leq C\left\|u_{0}\right\|_{H^{\delta}(M)}^{2} .
$$

Proof. We first calculate

$$
\begin{aligned}
\left\|\rho_{s} e^{i t P} u_{0}\right\|_{L_{T}^{2} H^{1}}^{2} \leq & C \int_{0}^{T} \int_{M}\left|\rho_{s}(P+1) e^{i t P} u_{0} \rho_{s} e^{-i t P} \overline{u_{0}}\right| d x d t \\
& +2 \int_{0}^{T} \int_{M}\left|\nabla \rho_{s}\right|\left|\nabla e^{i t P} u_{0}\right|\left|\rho_{s} e^{i t P} u_{0}\right| d x d t \\
& +\int_{0}^{T} \int_{M}\left|P\left(\rho_{s}\right)\right|\left|e^{i t P} u_{0}\right|\left|\rho_{s} e^{i t P} u_{0}\right| d x d t .
\end{aligned}
$$

Using

$$
\left|P\left(\rho_{s}\right)\right| \leq C\left|\nabla \rho_{s}\right| \leq C^{\prime}\left|\rho_{s}\right|
$$

and applying the Cauchy-Schwarz inequality yields

$$
\left\|\rho_{s} e^{i t P} u_{0}\right\|_{L_{T}^{2} H^{1}}^{2} \leq C\left\|u_{0}\right\|_{H^{2}} .
$$


Thus we have a linear operator bounded between complex interpolation spaces:

$$
\begin{aligned}
\rho_{s} e^{i t P} & : L^{2} \rightarrow L_{T}^{2} H^{1 / 2-\epsilon}, \\
& : H^{2} \rightarrow L_{T}^{2} H^{1} .
\end{aligned}
$$

Choosing $\epsilon=\delta / 4$ we have

$$
\begin{aligned}
\left\|\rho_{s} e^{i t P} u_{0}\right\|_{L_{T}^{2} H^{1 / 2}} & \leq C\left\|u_{0}\right\|_{H^{2 \epsilon /(1 / 2+\epsilon)}} \\
& \leq C\left\|u_{0}\right\|_{H^{\delta}} .
\end{aligned}
$$

\section{Proof of Theorem 2}

In this section we prove Theorem 2. The techniques of proof are borrowed partly from the study of energy decay for the damped wave equation on compact manifolds in Chr1, Leb, Hit, and [RT, among others, and partly from the techniques used in the study of the wave equation in exterior domains in [Mor, $\mathrm{MoPh}$, $\mathrm{MRS}$, and Vail.

For this section, we assume as in the statement of Theorem 2 that $M$ is equal to $\mathbb{R}^{n}$ outside a compact set, $n \geq 3$ is odd, and $\psi \in \mathcal{C}^{\infty}(M)$ satisfies (1.7). Consider the Hilbert spaces

$$
\mathcal{H}_{\epsilon}(M)=H^{1+\epsilon}(M) \times H^{\epsilon}(M),
$$

and the operator

$$
\mathcal{A}=\left(\begin{array}{cc}
0 & 1 \\
-\Delta_{g}+V & 0
\end{array}\right),
$$

where $V \in \mathcal{C}^{\infty}(M)$ now satisfies (1.6), which is an unbounded, operator on $\mathcal{H}_{0}$ with domain $\mathcal{H}_{1}$, satisfying $\mathcal{A}^{*}=\mathcal{A}^{t}$ as matrices. For an operator $\mathcal{P}: \mathcal{H}_{\epsilon} \rightarrow \mathcal{H}_{\epsilon^{\prime}}$, $f \in \mathcal{H}_{\epsilon}$, and $\psi$, we use the notation

$$
\psi \mathcal{P} f=\psi \operatorname{id}_{\mathcal{H}_{\epsilon^{\prime}} \rightarrow \mathcal{H}_{\epsilon^{\prime}}} \mathcal{P} f
$$

to mean multiplication by $\psi$ in each component.

To $\mathcal{A}$ we associate the semigroup $\mathcal{U}(t)=e^{i t \mathcal{A}}$, defined by the following evolution equation

$$
\left\{\begin{array}{l}
\left(D_{t}-\mathcal{A}\right) \mathcal{U}=0, \quad t>0 \\
\mathcal{U}(0)=\mathrm{id}_{\mathcal{H}_{0} \rightarrow \mathcal{H}_{0}}
\end{array}\right.
$$

We write

$$
f_{0}=\left(\begin{array}{l}
u_{0} \\
u_{1}
\end{array}\right) \in \mathcal{H}_{0}
$$

for our initial data. If we write the matrix representation of $\mathcal{U}$ as

$$
\mathcal{U}(t) f_{0}=\left(\begin{array}{cc}
V(t) & U(t) \\
D_{t} V(t) & D_{t} U(t)
\end{array}\right) f_{0},
$$

then

$$
u:=V(t) u_{0}+U(t) u_{1}
$$

solves (1.5). We can reformulate Theorem 2 to say we need to prove:

$$
\begin{aligned}
E_{\psi}(t) & =\left\|\psi \mathcal{U} f_{0}\right\|_{\mathcal{H}_{0}}^{2} \\
& \leq C e^{-t / C_{1}}\left\|f_{0}\right\|_{\mathcal{H}_{\epsilon}}^{2}
\end{aligned}
$$




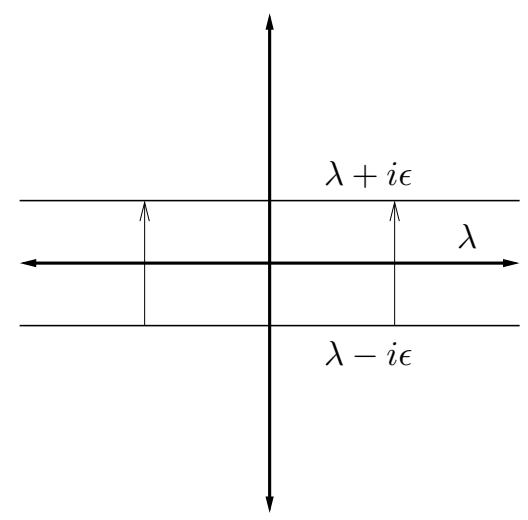

Figure 4. The holomorphic continuation of $\mathcal{R}(\lambda)$ to the nonphysical half-plane.

Formally, setting $\mathcal{U} f_{0} \equiv 0$ for $t<0$ and taking the Fourier transform in time in equation (4.1) suggests we should study the equation

$$
(\lambda-\mathcal{A}) \widehat{\mathcal{U}}(\lambda):=\hat{f}_{0}(\lambda) .
$$

Set $\mathcal{R}(\lambda)=(\mathcal{A}-\lambda)^{-1}$ and $R(\lambda)=\left(-\lambda^{2}-\Delta_{g}+V\right)^{-1}$.

Lemma 4.1. In matrix notation,

$$
\mathcal{R}(\lambda)=\left(\begin{array}{cc}
\lambda R(\lambda) & R(\lambda) \\
\lambda^{2} R(\lambda)+\mathrm{id} & \lambda R(\lambda)
\end{array}\right) .
$$

Further, if $n \geq 3$ is odd, there is a constant $C_{1}>0$ such that for each $\epsilon>0$,

$$
\|\psi \mathcal{R}(\lambda) \psi\|_{\mathcal{H}_{\epsilon} \rightarrow \mathcal{H}_{0}} \leq C_{\epsilon}
$$

for $\lambda \in \mathbb{R}+i\left[-C_{1}^{-1}, C_{1}^{-1}\right]$.

Proof. The first assertion follows by direct calculation. The second assertion follows from Remark 2.1 and Theorem 3

Now choose $\chi \in \mathcal{C}^{\infty}(\mathbb{R}), 0 \leq \chi \leq 1, \chi \equiv 0$ for $t \leq 0, \chi \equiv 1$ for $t \geq 1$. For

$$
f_{0}=\left(\begin{array}{l}
u_{0} \\
u_{1}
\end{array}\right) \in \mathcal{H}_{0}
$$

smooth with compact support, choose $\rho \in \mathcal{C}_{c}^{\infty}(M)$ so that

$$
\rho f_{0}=f_{0} \text {. }
$$

Set

$$
F_{1}(t)=\chi(t) \mathcal{U}(t) \rho f_{0}
$$

and calculate

$$
\begin{aligned}
\left(D_{t}-\mathcal{A}\right) F_{1}(t) & =-i \chi^{\prime}(t) \mathcal{U}(t) \rho f_{0} \\
& =\tilde{\psi} g_{1},
\end{aligned}
$$

where $\tilde{\psi} \in \mathcal{C}_{c}^{\infty}(M)$, supp $g_{1} \subset M \times[0,1]$, and

$$
\tilde{\psi} \mathcal{U}(t) f_{0}=\mathcal{U}(t) \rho f_{0} \text { on } \operatorname{supp} \chi^{\prime}(t)
$$


Such a $\tilde{\psi}$ exists since $\rho f_{0}$ has compact support and $\mathcal{U}(t) f_{0}$ has finite propagation speed.

Now

$$
\partial_{t}\left\langle\mathcal{U} f_{0}, \mathcal{U} f_{0}\right\rangle_{\mathcal{H}_{\epsilon}}=\left\langle i\left(\mathcal{A}+\mathcal{A}^{t}\right) \mathcal{U} f_{0}, \mathcal{U} f_{0}\right\rangle_{\mathcal{H}_{\epsilon}}
$$

has real part equal to 0, so using Gronwall's inequality,

$$
\begin{aligned}
\left\|g_{1}\right\|_{L^{2}\left([0,1] ; \mathcal{H}_{\epsilon}\right)} & \leq C\left\|\mathcal{U} f_{0}\right\|_{L^{2}\left([0,1] ; \mathcal{H}_{\epsilon}\right)} \\
& \leq C\left\|f_{0}\right\|_{\mathcal{H}_{\epsilon}} .
\end{aligned}
$$

Taking the Fourier transform in time of equation (4.3), we get

$$
(\lambda-\mathcal{A}) \widehat{F}_{1}(\lambda)=\tilde{\psi} \hat{g}_{1}(\lambda) .
$$

We have

$$
\begin{aligned}
\left\|e^{t / C_{1}} \psi F_{1}(t)\right\|_{L^{2}\left([0, \infty) ; \mathcal{H}_{0}\right)} & =\left\|\psi \widehat{F}_{1}\left(\lambda+i C_{1}^{-1}\right)\right\|_{L^{2}\left((-\infty, \infty) ; \mathcal{H}_{0}\right)} \\
& =\left\|\psi \mathcal{R}\left(\lambda+i C_{1}^{-1}\right) \tilde{\psi} \hat{g}_{1}\left(\lambda+i C_{1}^{-1}\right)\right\|_{L^{2}\left((-\infty, \infty) ; \mathcal{H}_{0}\right)} \\
& \leq C\left\|\hat{g}_{1}\left(\lambda+i C_{1}^{-1}\right)\right\|_{L^{2}\left((-\infty, \infty) ; \mathcal{H}_{\epsilon}\right)} \\
& =C\left\|g_{1}\right\|_{L^{2}\left([0,1] ; \mathcal{H}_{\epsilon}\right)} .
\end{aligned}
$$

Hence

$$
\left\|e^{t / C_{1}} \psi \mathcal{U}(t) f_{0}\right\|_{L^{2}\left([1, \infty) ; \mathcal{H}_{0}\right)} \leq C\left\|f_{0}\right\|_{\mathcal{H}_{\epsilon}} .
$$

Now to isolate the estimate near $T \geq 2$, choose $\chi_{2} \in \mathcal{C}^{\infty}(M), 0 \leq \chi_{2} \leq 1, \chi_{2} \equiv 0$ for $t \leq T-1, \chi_{2} \equiv 1$ for $t \geq T$. Set $F_{2}(t)=\chi_{2}(t) \mathcal{U}(t) f_{0}$, and calculate as before

$$
\begin{aligned}
\left(D_{t}-\mathcal{A}\right) V_{2} & =-i \chi_{2}^{\prime} \mathcal{U} f_{0} \\
& =\tilde{\psi} g_{2},
\end{aligned}
$$

where $\operatorname{supp} g_{2} \subset M \times[T-1, T]$. Set

$$
E_{2}(t)=\frac{1}{2}\left\|\psi V_{2}\right\|_{\mathcal{H}_{0}}^{2}
$$

and calculate

$$
E_{2}^{\prime}=\left\langle-i \psi \mathcal{A} V_{2}, \psi V_{2}\right\rangle+\left\langle\psi \tilde{\psi} g_{2}, \psi V_{2}\right\rangle
$$

which has real part bounded by $C E_{2}$. Since $E_{2}(T-1)=0$ and

$$
E_{2}(T)=E_{\psi}(T),
$$

Gronwall's inequality yields

$$
\begin{aligned}
E_{\psi}(T) & \leq C\left\|\psi \mathcal{U} f_{0}\right\|_{L^{2}\left([T-1, T] ; \mathcal{H}_{0}\right)}^{2} \\
& \leq C e^{-T / C_{1}}\left\|e^{t / C} \psi \mathcal{U} f_{0}\right\|_{L^{2}\left([1, \infty) ; \mathcal{H}_{\epsilon}\right)}^{2} \\
& \leq C e^{-T / C_{1}}\left\|f_{0}\right\|_{\mathcal{H}_{\epsilon}}^{2} .
\end{aligned}
$$




\section{Strichartz-type Inequalities}

In this section we prove several families of Strichartz-type inequalities and prove Proposition 1.3. The statements and proofs are mostly adaptations of similar inequalities in BGT2, so we leave out the proofs of these in the interest of space.

As in the statement of Proposition 1.3 we assume $M$ is asymptotically conic as defined in HTW and $V \in \mathcal{C}_{c}^{\infty}(M), V \geq 0$. The manifold $M$ admits the Sobolev embeddings recorded in the following proposition. For our notation, let

$$
W^{m, p}(M)\left(\operatorname{resp} . W_{0}^{m, p}(M)\right), m \in \mathbb{N}
$$

be the completion of $\mathcal{C}^{\infty}(M)$ (resp. $\left.\mathcal{C}_{c}^{\infty}(M)\right)$ with respect to the norm

$$
\|f\|_{W^{m, p}}^{p}=\sum_{|\alpha| \leq m}\left\|D^{\alpha} f\right\|_{L^{p}}^{p} .
$$

We define $W^{s, p}(M)$ and $W_{0}^{s, p}(M)$ for non-integer $s$ by interpolation. We use the convention

$$
H^{s}(M):=W^{s, 2}(M) \text {, and } H_{0}^{s}(M):=W_{0}^{s, 2}(M) .
$$

Let $H_{D}^{1}(M)$ denote the domain of $\left(1-\Delta_{g}\right)^{\frac{1}{2}}$ with Dirichlet boundary conditions if $\partial M \neq \emptyset$, so that $H_{D}^{1}(M)=H_{0}^{1}(M)$, and write $H_{D}^{s}(M)$ for the domain of $\left(1-\Delta_{g}\right)^{s / 2}$ with Dirichlet boundary conditions. Since $V \in \mathcal{C}_{c}^{\infty}(M)$, we may replace $\left(1-\Delta_{g}\right)^{\frac{1}{2}}$ with $(1+P)^{\frac{1}{2}}$ in the definitions, where $P=-\Delta_{g}+V(x)$. This results in equivalent Sobolev spaces with the addition $\left[P,(1+P)^{\frac{1}{2}}\right]=0$.

Proposition 5.1. We have the following continuous Sobolev embeddings:

$$
\begin{aligned}
& \text { (i) } H_{D}^{1}(M) \subset L^{p}(M), 2 \leq p \leq \frac{2 n}{n-2} \text {, or } p<\infty \text { for } n=2, \\
& \text { (ii) } H_{D}^{s}(M) \subset L^{p}(M), \frac{1}{2}=\frac{s}{n}+\frac{1}{p}, s \in[0,1), \\
& \text { (iii) } H_{D}^{s+1}(M) \subset W^{1, p}(M), \frac{1}{2}=\frac{s}{n}+\frac{1}{p}, s \in[0,1), \\
& \text { (iv) } W_{0}^{1, p}(M) \subset L^{q}(M), \frac{1}{p}=\frac{1}{n}+\frac{1}{q}, 1 \leq p<q<+\infty, \\
& \text { (v) } W_{0}^{s, p}(M) \subset L^{\infty}(M), s>\frac{n}{p}, p \geq 1 \\
& \text { (vi) } H_{D}^{s+1 / p}(M) \subset W^{s, q}(M), \frac{1}{p}+\frac{n}{q}=\frac{n}{2}, p \geq 2, s \in[0,1] .
\end{aligned}
$$

If we view $M \backslash U_{0}$ as a manifold with non-trapping geometry, we may apply the results of [HTW] to a solution of the Schrödinger equation away from the trapping region, resulting in perfect Strichartz estimates.

Proposition 5.2. For every $0<T \leq 1$ and each $\chi \in \mathcal{C}_{c}^{\infty}(M)$ satisfying $\chi \equiv 1$ near $U_{0} \cup \operatorname{supp} V$, there is a constant $C>0$ such that

$$
\|(1-\chi) u\|_{L^{p}([0, T]) W^{s, q}(M)} \leq C\left\|u_{0}\right\|_{H_{D}^{s}(M)},
$$

where $u=e^{-i t P} u_{0}, s \in[0,1]$, and $(p, q), p>2$ satisfy

$$
\frac{2}{p}+\frac{n}{q}=\frac{n}{2}
$$


Remark 5.3. In the sequel, wherever unambiguous, we will write

$$
L_{T}^{p} W^{s, q}:=L^{p}([0, T]) W^{s, q}(M)
$$

and

$$
H_{D}^{s}:=H_{D}^{s}(M) .
$$

Proposition 5.4. Let $u(t)=e^{-i t P} u_{0}$. For every $0<T \leq 1$ and $\epsilon>0$, there is a constant $C>0$ such that

$$
\|u\|_{L_{T}^{p} W^{s, q}} \leq C\left\|u_{0}\right\|_{H_{D}^{s+1 / p+\epsilon}},
$$

where $s \in[0,1]$ and $(p, q), p>2$ satisfy

$$
\frac{2}{p}+\frac{n}{q}=\frac{n}{2} .
$$

Remark 5.5. Proposition 5.4 represents the Strichartz estimates obtained by Burq-Gérard-Tzvetkov BGT2 in the case of non-trapping exterior domains with an $\epsilon>0$ loss due to the presence of the trapped orbit $\gamma$. Observe that (5.4) is weaker than the standard Euclidean Strichartz estimates in two ways, the loss of $1 / p$ derivatives from using Sobolev embeddings and the loss of $\epsilon$ derivatives from $\gamma$. When $\partial M=\emptyset$, we get the improvement given in Proposition 5.9.

Proposition 5.6. Let $u=e^{-i t P} u_{0}$ and

$$
v=\int_{0}^{t} e^{-i(t-\tau) P} f(\tau) d \tau .
$$

Then for each $0<T \leq 1$ and each $\delta>0$, there exists $C>0$ such that

$$
\|u\|_{L_{T}^{p} W^{s-\delta, q}} \leq C\left\|u_{0}\right\|_{H_{D}^{s}}
$$

and

$$
\|v\|_{L_{T}^{p} W^{s-\delta, q}} \leq C\|f\|_{L_{T}^{1} H_{D}^{s}},
$$

where $s \in[0,1]$ and $(p, q), p>2$ satisfy

$$
\frac{1}{p}+\frac{n}{q}=\frac{n}{2} .
$$

Remark 5.7. Proposition [5.6 is much weaker than the estimate suggested by scaling in Euclidean space, and as remarked in BGT2], is probably not optimal. We expect the $\delta>0$ loss to always hold due to the presence of $\gamma$, but the Euclidean scaling suggests the optimal estimate would replace $1 / p$ in 5.5 with $2 / p$ (see Proposition 5.9.

\section{Proposition 5.8. Let}

$$
v(t)=\int_{0}^{t} e^{-i(t-\tau) P} f(\tau) d \tau .
$$

For each $0<T \leq 1$ and each $\delta>0$, there is a constant $C>0$ such that

$$
\|v\|_{L_{T}^{p} W^{s-\delta, q}} \leq C\|f\|_{L_{T}^{p^{\prime} W^{s, q^{\prime}}}},
$$

where $p^{\prime}, q^{\prime}, p^{\prime} \in[1,2)$ are the duals of $p$ and $q$ satisfying (5.5), respectively, and satisfy

$$
\frac{1}{p^{\prime}}+\frac{n}{q^{\prime}}=\frac{n}{2}+1 .
$$


The next proposition is an improvement of Proposition 5.6 in the case $\partial M=\emptyset$.

Proposition 5.9. Suppose $(M, g)$ and $V$ satisfy the assumptions of Proposition 1.3. $u=e^{-i t P} u_{0}$

$$
v=\int_{0}^{t} e^{-i(t-\tau) P} f(\tau) d \tau,
$$

and in addition $\partial M=\emptyset$. Then for each $0<T \leq 1$ and each $\delta>0$, we have the estimates (5.3) and (5.4) for $s \in[0,1]$, where now $(p, q), p>2$ satisfy the Euclidean scaling

$$
\frac{2}{p}+\frac{n}{q}=\frac{n}{2}
$$

Proof. The idea of the proof is to use Proposition 5.2 to reduce the statement to a local question near the trapped orbit. Then we use a partition of unity and the local WKB construction from [BGT1] to get local in time Strichartz estimates for time on the scale of inverse frequency. We then sum up over frequencies and apply the local smoothing estimate to prove the Proposition. We remark this would also follow from [StTa, Theorem 4] and the local smoothing in Theorem 1]

Let $\chi$ be as in Proposition 5.2 and choose $\psi \in \mathcal{C}_{c}^{\infty}(\mathbb{R}), \psi \equiv 1$ near 1 and satisfying

$$
1 \leq \sum_{k \geq 0} \psi(r / k) \leq 2 \text { for } r \geq 0
$$

Choose also $\varphi \in \mathcal{C}_{c}^{\infty}(\mathbb{R}), \varphi \equiv 1$ on $\left[-c_{0}, c_{0}\right], \operatorname{supp} \varphi \subset\left[-2 c_{0}, 2 c_{0}\right]$ for $c_{0}>0$ small. Let

$$
w_{h}=\varphi(t / h) \chi(x) \psi\left(-h^{2} \Delta_{g}+h^{2} V(x)\right) u,
$$

which satisfies the equation

$$
\left\{\begin{array}{l}
\left(i \partial_{t}+\Delta_{g}-V(x)\right) w_{h}=\varphi\left[\Delta_{g}, \chi\right] \psi u+i \frac{1}{h} \varphi^{\prime} \chi \psi u \\
w_{h}(x, 0)=\chi \psi u_{0} .
\end{array}\right.
$$

Since $\varphi$ localizes to a timescale of size $h$, the semiclassical local WKB construction in BGT1] gives

$$
\left\|w_{h}\right\|_{L^{p} L^{q}} \leq C\left\|\varphi\left[\Delta_{g}, \chi\right] \psi u+i \frac{1}{h} \varphi^{\prime} \chi \psi u\right\|_{L^{1} L^{2}},
$$

with $(p, q), p>2$ satisfying (5.7).

Choose $\tilde{\varphi} \in \mathcal{C}_{c}^{\infty}(\mathbb{R})$ and $\tilde{\chi} \in \mathcal{C}_{c}^{\infty}(M)$ satisfying $\tilde{\varphi} \equiv 1$ on $\operatorname{supp} \varphi$ and $\tilde{\chi} \equiv 1$ on $\operatorname{supp} \chi$. Applying Hölder's inequality in time and exchanging one derivative for $h^{-1}$ on the support of $\psi$ yields

$$
\left\|w_{h}\right\|_{L^{p} L^{q}} \leq C h^{1 / 2}\left\|h^{-1} \tilde{\varphi} \tilde{\chi} \psi u\right\|_{L^{2} L^{2}} .
$$

Exchanging one half derivative with $h^{-1 / 2}$ we obtain

$$
\left\|w_{h}\right\|_{L^{p} L^{q}} \leq C\|\tilde{\varphi} \tilde{\chi} \psi u\|_{L^{2} H^{1 / 2}},
$$

and summing in $h=1 / k$ we get

$$
\|\chi u\|_{L^{p} L^{q}} \leq C\|\tilde{\chi} u\|_{L^{2} H^{1 / 2}}
$$

which after a time truncation and an application of Lemma 3.3 proves the Proposition for $u$. Finally, an application of the Christ-Kiselev lemma ChKi] proves the proposition for $v$. 
Remark 5.10. We remark that the frequency localization Strichartz estimates in the proof of Proposition 5.9 have been obtained independently by Schlag-SofferStaubach SSS in the case $M$ is a 2-dimensional asymptotically conic surface of revolution and the initial data is radial. This includes, for example, the catenoid, which admits a single closed hyperbolic geodesic. Combined with Theorem 1, this again recovers Proposition 5.9.

Proof of Proposition 1.3. The proof of Proposition 1.3 is a slight modification of the proof of Proposition 3.1 in [BGT1, but we include it here in the interest of completeness. First we assume $\partial M \neq \emptyset$. Fix $s$ satisfying 1.12 and choose $p>$ $\max \{2 \beta-2,2\}$ satisfying

$$
s>\frac{n}{2}-\frac{1}{p}+\delta \geq \frac{n}{2}-\frac{1}{\max \{2 \beta-2,2\}}
$$

for some $\delta>0$. Set $\sigma=s-\delta$ and

$$
Y_{T}=C\left([-T, T] ; H_{D}^{s}(M)\right) \cap L^{p}\left([-T, T] ; W_{0}^{\sigma, q}(M)\right)
$$

for

$$
\frac{1}{p}+\frac{n}{q}=\frac{n}{2}
$$

equipped with the norm

$$
\|u\|_{Y_{T}}=\max _{|t| \leq T}\|u(t)\|_{H_{D}^{s}(M)}+\|u\|_{L_{T}^{p} W^{\sigma, q}} .
$$

Let $\Phi$ be the nonlinear functional

$$
\Phi(u)=e^{-i t P} u_{0}-i \int_{0}^{t} e^{-i(t-\tau) P} F(u(\tau)) d \tau .
$$

If we can show that $\Phi: Y_{T} \rightarrow Y_{T}$ and is a contraction on a ball in $Y_{T}$ centered at 0 for sufficiently small $T>0$, this will prove the first assertion of the Proposition, along with the Sobolev embedding

$$
W_{0}^{\sigma, q}(M) \subset L^{\infty}(M),
$$

since $\sigma>n / q$. From Proposition 5.6, we bound the $W^{\sigma}$ part of the $Y_{T}$ norm by the $H_{D}^{s}$ norm, giving

$$
\begin{aligned}
\|\Phi(u)\|_{Y_{T}} & \leq C\left(\left\|u_{0}\right\|_{H_{D}^{s}}+\int_{-T}^{T}\|F(u(\tau))\|_{H_{D}^{s}} d \tau\right) \\
& \left.\leq C\left(\left\|u_{0}\right\|_{H_{D}^{s}}+\int_{-T}^{T}\|(1+|u(\tau)|)\|_{L^{\infty}}^{2 \beta-2}\right)\|u(\tau)\|_{H_{D}^{s}} d \tau\right),
\end{aligned}
$$

where the last inequality follows by our assumptions on the structure of $F$. Applying Hölder's inequality in time with $\tilde{p}=p /(2 \beta-2)$ and

$$
\frac{1}{\tilde{q}}+\frac{1}{\tilde{p}}=1
$$

gives

$$
\left.\|\Phi(u)\|_{Y_{T}} \leq C\left(\left\|u_{0}\right\|_{H_{D}^{s}}+T^{\gamma}\|u\|_{L_{T}^{\infty} H_{D}^{s}}\|(1+|u|)\|_{L_{T}^{p} L^{\infty}}^{2 \beta-2}\right)\right)
$$

where $\gamma=1 / \tilde{q}>0$. Thus

$$
\|\Phi(u)\|_{Y_{T}} \leq C\left(\left\|u_{0}\right\|_{H_{D}^{s}}+T^{\gamma}\left(\|u\|_{Y_{T}}+\|u\|_{Y_{T}}^{2 \beta}\right)\right) .
$$


Similarly, we have for $u, v \in Y_{T}$,

$$
\begin{aligned}
& \|\Phi(u)-\Phi(v)\|_{Y_{T}} \leq \\
& \left.\quad \leq C T^{\gamma}\|u-v\|_{L_{T}^{\infty} H_{D}^{s}}\|(1+|u|)\|_{L_{T}^{p} L^{\infty}}^{2 \beta-2}+\|(1+|v|)\|_{L_{T}^{p} L^{\infty}}^{2 \beta-2}\right) \\
& \left.\quad \leq C T^{\gamma}\|u-v\|_{Y_{T}}\|(1+|u|)\|_{Y_{T}}^{2 \beta-2}+\|(1+|v|)\|_{Y_{T}}^{2 \beta-2}\right),
\end{aligned}
$$

which is a contraction for sufficiently small $T$. This concludes the proof of the first assertion in the Proposition.

To get the second assertion, we observe from 5.9 and the definition of $Y_{T}$, if $u$ and $v$ are two solutions to (1.11) with initial data $u_{0}$ and $u_{1}$ respectively, so

$$
\widetilde{\Phi}(v)=e^{-i t P} u_{1}-i \int_{0}^{t} e^{-i(t-\tau) P} F(v(\tau)) d \tau,
$$

we have

$$
\begin{aligned}
\max _{|t| \leq T} \| u(t) & -v(t) \|_{H_{D}^{s}} \\
= & \max _{|t| \leq T}\|\Phi(u)(t)-\widetilde{\Phi}(v)(t)\|_{H_{D}^{s}} \\
\leq C \quad\left(\left\|u_{0}-u_{1}\right\|_{H_{D}^{s}}\right. & \\
& \left.\left.\quad+T^{\gamma} \max _{|t| \leq T}\|u(t)-v(t)\|_{H_{D}^{s}}\|(1+|u|)\|_{L_{T}^{p} L^{\infty}}^{2 \beta-2}+\|(1+|v|)\|_{L_{T}^{p} L^{\infty}}^{\beta \beta-2}\right)\right),
\end{aligned}
$$

which, for $T>0$ sufficiently small gives the Lipschitz continuity.

In the case $\partial M=\emptyset$, we have the improved Strichartz estimates given in Proposition 5.9. Hence we can take $s$ and $p$ satisfying $p>\max \{2 \beta-2,2\}$ and

$$
s>\frac{n}{2}-\frac{2}{p}+\delta \geq \frac{n}{2}-\frac{2}{\max \{2 \beta-2,2\}}
$$

for $\delta>0$. Then $\sigma=s-\delta>q / n$ and the preceding argument holds with these modifications.

The proof of Corollary 1.4 now follows from the standard global well-posedness arguments from, for example, [Caz, Chapter 6].

\section{REFERENCES}

[BrPe] Bruneau, V. and Petkov, V. Semiclassical Resolvent Estimates for Trapping Perturbations. Comm. Math. Phys. 213, 2000, p. 413-432.

[Bur1] Burq, N. Décroissance de l'énergie locale de l'équation des ondes pour le problème extérieur et absence de résonance au voisinage du réel. Acta Math. 180, 1998, p. 1-29.

[Bur2] Burq, N. Smoothing Effect for Schrödinger Boundary Value Problems. Duke Math. Journal. 123, No. 2, 2004, p. 403-427.

[BGT1] Burq, N. Gérard, P., and Tzvetkov, N. Strichartz Inequalities and the Nonlinear Schrödinger Equation on Compact Manifolds. Amer. J. Math. 126, No. 3, 2004, p. 569-605.

[BGT2] Burq, N., Gérard, P., and Tzvetkov, N. On Nonlinear Schrödinger Equations in Exterior Domains. Ann. I H. Poincaré. 21, 2004, p. 295-318.

[CPV] Cardoso, F., Popov, G., and Vodev, G. Semi-classical Resolvent Estimates for the Schrödinger Operator on Non-compact Complete Riemannian Manifolds. Bull. Braz. Math Soc. 35, No. 3, 2004, p. 333-344. 
[Caz] Cazenave, T. Semilinear Schrödinger Equations. Courant Lecture Notes in Mathematics, AMS, 2003.

[ChKi] Christ, M. And Kiselev, A. Maximal Functions Associated to Fiiltrations. J. Funct. Anal. 179, No. 2, 2001, p. 409-425.

[Chr1] Christianson, H. Semiclassical Non-concentration near Hyperbolic Orbits. to appear, J. Funct. Anal.. http://www.math.berkeley.edu/ hans/papers/nc.pdf.

[Chr2] Christianson, H. Quantum Monodromy and Non-concentration near Semi-hyperbolic Orbits. in preparation.

[Doi] Dor, S.-I. Smoothing effects of Schrödinger Evolution Groups on Riemannian Manifolds. Duke Mathematical Journal. 82, No. 3, 1996, p. 679-706.

[EvZw] Evans, L.C. And Zworski, M. Lectures on Semiclassical Analysis. http://math.berkeley.edu/ evans/semiclassical.pdf

[HTW] Hassell, A., TaO, T., AND Wunsch, J. Sharp Strichartz Estimates on Non-trapping Asymptotically Conic Manifolds. preprint. 2004. http://www.arxiv.org/pdf/math.AP/0408273

[Hit] HitrIK, M. Eigenfrequencies and Expansions for Damped Wave Equations. Methods and Applications of Analysis. 10, No. 4, 2003, p. 543-564.

[Leb] Lebeau, G. Equation des Ondes Amorties. Algebraic and Geometric Methods in Mathematical Physics, A Boutet de Monvel and V. Marchenko (eds.) Kluwer Academic Publishers, Netherlands, 1996. 73-109.

[Mel] Melrose, R. Spectral and scattering theory for the Laplacian on asymptotically Euclidian spaces. Spectral and scattering theory, p. 85-130, Lecture Notes in Pure and Appl. Math. 161, Dekker, New York, 1994.

[MeSo1] Metcalfe, J. And Sogge, C. Global existence for Dirichlet-wave equations with quadratic nonlinearities in high dimensions. Math. Ann. 336 (2006) p. 391-420.

[MeSo2] Metcalfe, J. And Sogge, C. Hyperbolic trapped rays and global existence of quasilinear wave equations. Invent. Math. 159 (2005), No. 1, p. 75-117.

[Mor] Morawetz, C. The decay of solutions of the exterior initial-boundary value problem for the wave equation. Comm. Pure Appl. Math. 14 (1961) p. 561-568.

[MoPh] Morawetz, C. And Phillips, R. The exponential decay of solutions of the wave equation in the exterior of a star-shaped obstacle. Bull. Amer. Math. Soc. 68 (1962) p. 593-595.

[MrS] Morawetz, C., Ralston, J., and Strauss, W. Decay of solutions of the wave equation outside nontrapping obstacles. Comm. Pure Appl. Math. 30, No. 4, (1977) p. 447-508.

[PeZw] Petkov, V. And Zworski, M. Semi-classical Estimates on the Scattering Determinant. Ann. I. H. Poincaré. 2, 2001, p. 675-711.

[Ral] RALson, J. Trapped rays in spherically symmetric media and poles of the scattering matrix. Comm. Pure Appl. Math. 24 (1971), p. 571-582.

[RT] Rauch, J. And TAYlor, M. Decay of Solutions to Nondissipative Hyperbolic Systems on Compact Manifolds. Comm. Pure Appl. Math. 28, No. 4, 1975, p. 501-523.

[SSS] Schlag, W., Soffer, A., And Staubach, W. Decay estimates for the Schroedinger evolution on asymptotically conic surfaces of revolution I. preprint, http://www.arxiv.org/pdf/math.AP/0608075

[Sjö] SJÖSTRAND, J. Lectures on Resonances. http://www.math.polytechnique.fr/ sjoestrand/CoursgbgWeb.pdf

[StTa] Staffilani, G. And Tataru, D. Strichartz Estimates for a Schrödinger Operator with Nonsmooth Coefficients. Comm. Partial Differential Equations. 27, No. 5-6, 2002, p. $1337-1372$.

[Vai] Vainberg, B. Asymptotic methods in equations of mathematical physics. Translated from the Russian by E. Primrose. Gordon \& Breach Science Publishers, New York, 1989.

[Vod] Vodev, G. Local Energy Decay of Solutions to the Wave Equation for Nontrapping Metrics. Ark. Mat. 42, 2004, p. 379-397.

Department of Mathematics, University of California, Berkeley, CA 94720 USA

E-mail address: hans@math.berkeley.edu 